\title{
Central retinal artery occlusion following cosmetic blepharoplasty
}

\author{
PHILLIP W. KELLY' AND DONALD R. MAY' \\ From the ${ }^{1}$ Ophthalmology Service, Wilford Hall USAF Medical Center, Lackland Air Force Base, \\ San Antonio, Texas, and the ${ }^{2}$ Department of Ophthalmology, School of Medicine, \\ University of California, Davis, California 95616, USA
}

SUMMARY A case is presented of a 70-year-old white male who underwent a bilateral cosmetic lower lid blepharoplasty with fat removal. He subsequently developed a right orbital haemorrhage which resulted in the occlusion of the central retinal artery. The patient had no light perception with his right eye for approximately 1 hour, but vision was eventually restored to 20/20 after emergency therapeutic measures. There are many possible causes of visual loss following blepharoplasty, with orbital haemorrhage being the most common. Visual loss secondary to blepharoplasty can be prevented in the preoperative, intraoperative, and postoperative phases of surgery. Blindness as a complication of cosmetic blepharoplasty can be reversible if recognised early and treated appropriately. These facts must be kept in mind by all surgeons who perform blepharoplasties.

Blindness following cosmetic blepharoplasty is rare. According to DeMere's survey of $\mathbf{3 0 0 0}$ physicians performing blepharoplasties ${ }^{1}$ the occurrence was $0.04 \%$ or 1 case per 2500 operations. In 1969 Smith $^{2}$ reported that visual loss following blepharoplasty was rarely encountered as a complication of blepharoplasty. Castanares ${ }^{3}$ reported that he had seen only 1 case of blindness following blepharoplasty, but had seen at least 40 cases of unilateral partial blindness during his 25 years of practice. Jafek ${ }^{4}$ reported one case of unilateral blindness attributed to an intraorbital haemorrhage following blepharoplasty with fat excision. His review of the medical literature revealed no case reports for such an occurrence over the previous 5 years. Putterman ${ }^{5}$ reported the case of a patient who suffered complete unilateral blindness following blepharoplasty with fat excision. With therapeutic intervention the affected eye of this patient was returned to its preoperative visual acuity with no apparent sequelae.

Hueston and Heinze ${ }^{6}$ discussed the early diagnosis and surgical reversal of a case of blindness following blepharoplasty. They later reported a similar case of blindness reversed by wound decompression. ${ }^{7}$ They concluded that outpatient blepharoplasty procedures were 'intrinsically unsafe' and recommended close inpatient supervision postoperatively.

Correspondence to Major Phillip W. Kelly, USAF MC, Ophthalmology Service/SGHSE, USAF Regional Hospital, Sheppard (ATC), Sheppard Air Force Base, Texas 76311, USA.
Blindness following orbital surgery other than blepharoplasty was reported by Long and Ellis ${ }^{8}$ in 1971. Eight cases were described which followed orbital exposure by Berke-Krönlein technique. Blindness has also been described following reduction of fractured zygoma, ${ }^{9}$ the repair of orbital floor fractures, ${ }^{10}$ retinal detachment procedures, ${ }^{11}$ and neurosurgical procedures. ${ }^{12}$

The most recent literature review and discussion of blindness following blepharoplasty was by Waller. ${ }^{13} \mathrm{He}$ concluded that blindness is a rare but real complication of blepharoplasty which must be considered by the physician performing this operation.

\section{Case report}

A 70-year-old white male underwent bilateral cosmetic upper lid blepharoplasties with fat removal. Six weeks later he was scheduled to undergo cosmetic bilateral lower lid blepharoplasties, also with fat excision. His preoperative examination included a visual acuity of 20/20 in both eyes and was otherwise completely normal except for dermatochalasis of both lower lids. He had no history of hypertension or any other medical problem and was receiving no medication.

The procedure performed was a standard bilateral lower lid blepharoplasty under local anaesthesia as described in the AAOO manual on ophthalmic plastic surgery. ${ }^{14}$ Fat excision was performed without 


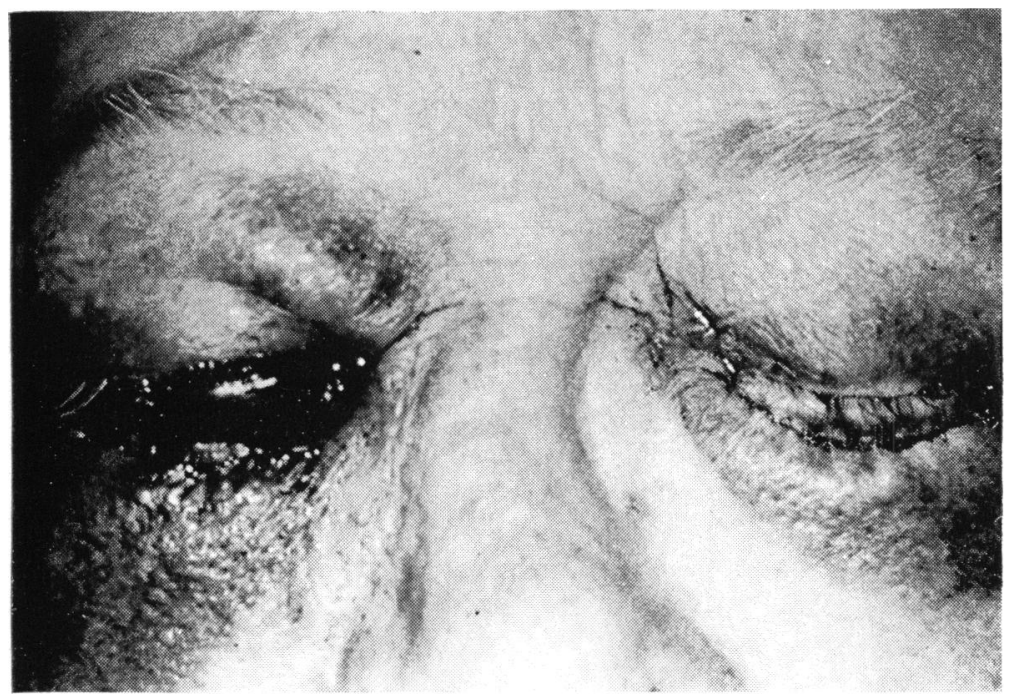

Fig. 1 Four hours following bilateral lower lid blepharoplasties with fat excision. There has been a right orbital haemorrhage with proptosis, subconjunctival haemorrhage, chemosis, and periorbital oedema. Vision is no light perception with the right eye and 20/20 with the left.

difficulty. The fat presented easily and haemostasis was achieved by cauterising the cut end of fat before releasing the haemostat. The orbital septum was not sutured. Haemostasis appeared adequate at the end of the procedure. A small pressure patch was placed over both infraorbital rims, leaving the eyes uncovered. The patient was instructed to remove the patches on arriving home and to apply ice packs intermittently over both lower lids.

The patient began to notice right orbital pain and significant swelling of the right lower lid 2 hours after surgery. He returned to the ophthalmology clinic about 4 hours after surgery. He stated that he had been unable to see light with his right eye for more than 1 hour before returning to the clinic. He had also experienced severe pain of his right eye during that time.

The initial evaluation revealed marked proptosis of the right eye with extensive subconjunctival haemorrhage, chemosis, limited motility, and periorbital oedema (Fig. 1). The right eye was hard to finger palpation, and the intraocular pressure was over $60 \mathrm{mmHg}$ measured with a Schiøtz tonometer with a $15 \mathrm{~g}$ weight. The right eye had no light perception. The right pupil was not reactive to light but was reactive consensually to light to the left eye and to a near stimulus. Direct and indirect ophthalmoscopic examination of the right eye revealed no perfusion of blood through the central retinal artery or its branches and stagnation of blood in the venous system.

Immediate therapeutic measures were instituted. Initially a right lateral canthotomy was performed. The right lower lid suture line was then opened and approximately $10 \mathrm{ml}$ of blood under pressure was released. A $3 \mathrm{ml}$ retrobulbar injection was given consisting of equal parts of lidocaine $\mathrm{HCl} 2 \%$, hyaluronidase, and aminophylline. An additional $2 \mathrm{ml}$ of hyaluronidase was injected into the swollen right lower lid. Intermittent digital massage was applied to the right eye. An intravenous drip was started and $500 \mathrm{mg}$ of acetazolamide was given rapidly followed by $25 \mathrm{~g}$ of mannitol over 3 minutes. No additional mannitol was given. An additional $250 \mathrm{mg}$ of acetazolamide was given intravenously every 6 hours for the next 24 hours. Bag rebreathing was started with the patient in the supine position until carbogen $\left(5 \% \mathrm{CO}_{2}, 95 \% \mathrm{O}_{2}\right)$ became available. Carbogen was administered with continuous monitoring of the patient's pulse, respirations, and blood pressure for 10 minutes every hour for 12 hours.

After these measures were performed 15 minutes had passed and the intraocular pressure was 38 $\mathrm{mmHg}$ with a Schiøtz tonometer with a $10 \mathrm{~g}$ weight. Ophthalmoscopy revealed perfusion of the entire retina at that time without apparent occlusion of any portion of the retinal artery system. Within 30 minutes the temporal half of the patient's right visual field returned increasing to a full visual field to confrontation within 1 hour after treatment was begun. At that time the patient's vision with his right eye was 20/200 with a near card.

Gentamicin $80 \mathrm{mg}$ intramuscularly every 8 hours and methicillin sodium $2 \mathrm{mg}$ intravenously every 6 hours were started and continued for 72 hours. After drainage from the lower lid wound ceased at 48 hours, a retroauricular skin graft was used to close the wound.

The visual acuity in the right eye improved to 
Fig. 2 Ten months after the central retinal artery occlusion. $A$ satisfactory cosmetic result has been achieved. Vision is 20/20 with both eyes.

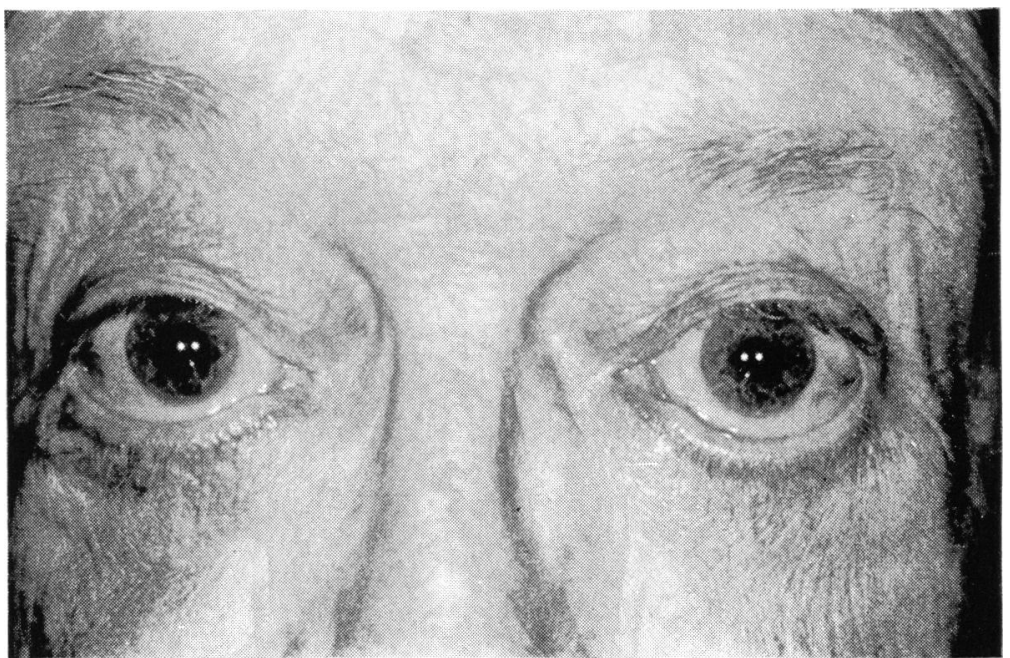

$20 / 60$ by 48 hours and to 20/20 within 72 hours of treatment. Visual field perimetry in the right eye showed slight constriction of the central isoptre at 24 hours, but reverted to normal within 48 hours. The patient's vision was 20/20 in the right eye with a full visual field 10 months after the central retinal artery occlusion. A good cosmetic result was also achieved on his right lower lid (Fig. 2).

\section{Discussion}

Various mechanisms have been proposed as the cause of visual loss following blepharoplasty. Most authors feel that increased intraorbital pressure is a major factor, whether it is a result of orbital haemorrhage or oedema as a result of operative manipulation. Hepler et al.$^{15}$ felt that sudden or complete loss of vision following blepharoplasty was caused by (1) orbital haemorrhage causing increased orbital pressure which compromised the vascular circulation within the eye; (2) acute angle-closure glaucoma in susceptible individuals; or (3) optic nerve ischaemia, possibly due to orbital manipulation and/or pressure or secondary systemic hypotension from preoperative medications. Also all the cases of visual loss following blepharoplasty which he and others $^{13}$ reported have been associated with fat removal at the time of surgery.

Putterman ${ }^{5}$ proposed additional causes for orbital haemorrhages: (1) eyelid neovascularisation secondary to retinal detachment repairs; and (2) rebound vasodilatation from epinephrine-constricted vessels escaping cautery during surgery. Another commonly reported cause of orbital haemorrhage is excessive tugging on orbital fat during excision. Inadequate haemostasis of excised fat by failure to adequately cauterise the cut ends can also lead to orbital haemorrhage. Other contributing causes to orbital haemorrhage are bleeding diatheses or severe arteriosclerosis.

The usual mechanism of visual loss is increased intraorbital pressure producing a central retinal artery and/or vein occlusion, or causing direct pressure on the optic nerve with subsegment occlusion of its vascular supply. Total vascular insufficiency for 60-120 minutes produces permanent loss of vision. ${ }^{7}$ A lesser duration of occlusion leads to variable visual recovery. Clinically the signs and symptoms of orbital haemorrhage are an acute onset of orbital pain with an increased intraorbital pressure manifested by proptosis, lid ecchymosis, increased intraocular pressure, limited extraocular muscle movement, and a dilated, unresponsive pupil. Visual acuity is usually reduced but can range from $20 / 20$ to no light perception. The clinician should be familiar with these signs and symptoms so that he may act quickly and definitively should they appear.

The treatment of orbital haemorrhage as shown by the above signs and symptoms varies according to the author. If no visual loss has occurred and vision does not appear threatened, close observation of visual acuity, intraocular pressure, and fundus vessel perfusion may be all that is required. However, when evidence of acute or impending decreased retinal perfusion occurs, immediate therapy must be initiated. If the surgeon caring for the patient is not an ophthalmologist, immediate assistance from a qualified ophthalmologist should be obtained while emergency measures are being instituted.

A lateral canthotomy can be performed easily to decrease intraorbital pressure. The surgical wound 
should also be opened and the haematoma evacuated. A search for the bleeding site should be performed.

An intravenous drip should be started and $20 \%$ mannitol solution should be given as a bolus of $12.5 \mathrm{~g}(62.5 \mathrm{ml})$ over an initial 3-5 minute interval, followed by a slower administration of additional mannitol solution to achieve a total dose of $1 \mathrm{~g} / \mathrm{kg}$ of body weight over the next 30 minutes. ${ }^{16}$ Intravenous acetazolamide $(500 \mathrm{mg})$ is given as an intravenous push bolus with an additional $250 \mathrm{mg}$ given intravenously every 6 hours for at least the first 24 hours. Digital ocular massage may be helpful to decrease intraocular pressure while awaiting action of the intravenous osmotic agent. The patient should be placed horizontally and made to rebreathe in a small paper bag (a classic lunch bag size is best) until a mixture of $95 \% \mathrm{O}_{2} / 5 \% \mathrm{CO}_{2}$ (carbogen) can be obtained for intermittent breathing. Carbogen is available from most pulmonary therapy laboratories. The injection of retrobulbar xylocaine and aminophylline can be performed to promote dilation of the central retinal artery. The efficacy of this treatment has not been definitively proved and is not universally recommended. Hyaluronidase is added to promote diffusion of the xylocaine and aminophylline through the orbital tissues. The hyaluronidase also greatly enhances the reabsorption of fluid from the orbital tissues. ${ }^{17}$

Paracentesis of the anterior chamber has been advocated to reduce intraocular pressure, ${ }^{16}$ but this is a hazardous procedure and should be performed only by an ophthalmologist experienced in the technique..$^{18}$ Intraocular haemorrhage, cataract formation, and iris prolapse are only 3 of the possible complications which can result from a paracentesis.

Systemic antibiotics should be started after opening of a surgical wound for the evacuation of a haemorrhage. The wound should be closed again only after drainage has ceased.

During the acute treatment phase the patient should be carefully monitored with visual acuities, pupillary response, funduscopy, and confrontation visual fields to assess the response to therapy. After the immediate therapy has ceased the visual acuity and visual fields should be examined and recorded.

The prevention of orbital haemorrhage with subsequent visual loss can be grouped into 3 categories: preoperative, intraoperative, and postoperative. ${ }^{19}$

\section{(1) PREOPERATIVE}

Moser et al. ${ }^{20}$ reported nonsurgically related postoperative blindness following blepharoplasty surgery. They emphasised the need for preoperative examination and recording of visual acuity and other unassociated ocular pathology. DeMere et al. ${ }^{1}$ found that adequate eye examinations were performed in only $50 \%$ of patients undergoing eyelid surgery. This ranged from a high of $90 \%$ in ophthalmologists to $15 \%$ for plastic surgeons. Amblyopia is present in about $2 \%$ of our population, and coexistent eye disease in patients undergoing eyelid surgery is a distinct possibility which must be ruled out, for example, cataract, glaucoma, old trauma or scar, or retinal detachment. Patients often do not realise that one eye is blind until the opposite eye is closed or covered. Discovering this immediately after surgery, even though it was pre-existent, causes the patient to relate the decreased visual acuity to the surgery itself.

A good drug history should be obtained with particular emphasis on vasodilators, aspirin, warfarin sodium, and other blood coagulation altering agents. An evaluation for arteriosclerosis and hypertension should also be performed. Any eye abnormalities should be noted and recorded if possible with photographs. One should rule out thyroid or renal disease as causes for lid abnormalities. ${ }^{19} \mathrm{~A}$ surgeon should be hesitant to perform blepharoplasty with fat removal in a one-eyed patient unless it is definitely indicated. A history of a true allergy to local anaesthetics obviates their use during blepharoplasty and general anaesthesia is indicated instead.

\section{(2) INTRAOPERATIVE}

During the operative procedure care must be given to meticulous dissection, atraumatic tissue handling, and complete haemostasis. Undue traction on the orbital contents, especially during fat removal, can lead to orbital haemorrhage. Fat removal must be limited to that fat which presents itself through the orbital septum with only light pressure on the globe. The orbital septum should not be excessively opened to obtain orbital fat. When excising orbital fat, a clamp should be placed on the fat, the fat excised with scissors (to leave a larger stump on the clamp for cauterisation), and cauterisation carried out with a hot wire or bipolar cautery. Cautery should be used with care anterior to the orbital septum and never intraorbitally. Electrocoagulation by diathermy is not thought to be adequate for haemostasis. When the fat has been cauterised, the clamp should then be opened and the distal stump of fat examined for bleeding with additional cautery as necessary. Before closing the wound a dry field is essential to avoid complications. If epinephrine is used with the local anaesthetic, care must be exercised that postoperative rebound vascular dilatation does not occur in a previously dry field. 
Avoidance of inadvertent excessive pressure on the globe should be practised. Hollenhorst et al. ${ }^{12}$ reported that central retinal artery occlusions can occur during cervical neurosurgical procedures as a result of the head rest causing inadvertent ocular pressure. If an eye is covered during any surgical procedure, a protective metal shield must be properly placed over the eye to prevent inadvertent pressure.

\section{(3) POSTOPERATIVE}

Postoperative pressure dressings are mentioned only to be condemned. Pressure increases as reported by Jarrett and Brockhurst ${ }^{11}$ do occur with elastic bandages over the eyes and can contribute to vascular compromise. Pressure dressings obscure observation needed to detect decreasing visual acuity, increasing intraocular pressure, and signs of orbital haemorrhage or other increases in orbital pressure. If an orbital haemorrhage occurs, pressure dressings raise the intraorbital pressure to much higher levels than if no patch were present. Many authors feel that blepharoplasty with fat removal should not be done unless careful postoperative observation is possible, meaning hospital admission in most cases.

During the first 2 hours postoperatively visual acuity assessments and observation for pain or proptosis should be performed every 10 minutes. The intervals can be lengthened to every 4 hours from 2 to 24 hours after surgery. The examination must be performed by qualified medical observers. A gauze pad soaked in iced or chilled saline can be loosely applied over the eyes for the first 24 hours to decrease swelling.

Should signs or symptoms of an orbital haemorrhage occur, the surgeon should be contacted immediately. If decreasing visual acuity is found secondary to the suspected orbital haemorrhage, emergency treatment measures should be immediately instituted as outlined above. If visual loss is reversed, careful monitoring of the patient's visual status is necessary including visual acuity, pupillary reaction, fundus examination, and visual fields until the patient's condition is entirely stable.

Dr John W. Shore and Dr Hal C. Hudson, Captain Marsha Yeager Sherry, and Lieut. Nancy Wylie provided invaluable assistance during the emergency care of this patient.

\section{References}

1 DeMere M, Wood T, Austin W. Eye complications with blepharoplasty or other eyelid surgery. A national survey. Plast Reconstr Surg 1974; 53: 634-7.

2 Smith B. Postsurgical complications of cosmetic blepharoplasty. Trans Am Acad Ophthalmol Otolaryngol 1969; 73: $1162-4$.

3 Castanares S. Eyelid Plasty. In: Goldwyn RM, ed. The Unfavorable Result in Plastic Surgery: Avoidance and Treatment. Boston: Little, Brown, 1972: 267.

4 Jafek BW, Kreiger AE, Morledge D. Proceedings: Blindness following blepharoplasty. Arch Otolaryngol 1973; 98: 366-9.

5 Putterman AM. Temporary blindness after cosmetic blepharoplasty. Am J Ophthalmol 1975; 80: 1081-3.

6 Hueston JT, Heinze JB. Successful early relief of blindness occurring after blepharoplasty. Case report. Plast Reconstr Surg 1974; 53: 588-92.

7 Hueston JT, Heinze JB. A second case of relief of blindness following blepharoplasty: Case Report. Plast Reconstr Surg 1977; 59: 430-1.

8 Long JC, Ellis PP. Total unilateral visual loss following orbital surgery. Am J Ophthalmol 1971 ; 71: 218-20.

9 Varley EWB, Holt-Wilson AD, Watson PG. Acute retinal arterial occlusion following reduction of a fractured zygoma and its successful treatment. $\mathrm{Br} J$ Oral Surg 1968; 6: 31-6.

10 Nicholson DH, Guzak SV Jr. Visual loss complicating repair of orbital floor fractures. Arch Ophthalmol 1971; 86: $369-75$.

11 Jarrett WH, Brockhurst RJ. Unexplained blindness and optic atrophy following retinal detachment surgery. Arch Ophthalmol 1965; 73: 782-91.

12 Hollenhorst RW, Svien HJ, Benoit CF. Unilateral blindness occurring during anesthesia for neurosurgical operations. Arch Ophthalmol 1954; 52: 819-30.

13 Waller $R$. Is blindness a realistic complication in blepharoplasty procedures? Ophthalmology 1978; 85: $730-5$.

14 Silver B. Ophthalmic Plastic Surgery. 3rd ed. Rochester: American Academy of Ophthalmology and Otolaryngology, 1977.

15 Hepler RS, Sugimura GI, Straatsma BR. On the occurrence of blindness in association with blepharoplasty. Plast Reconstr Surg 1976; 57: 233-5.

16 Hartley JM Jr, Lester JC, Schatten WE. Acute retrobulbar haemorrhage during elective blepharoplasty: its pathophysiology and management. Plast Reconstr Surg 1973; 52: 8-12.

17 May DR. Hyaluronidase in the treatment of orbital haemorrhage. In preparation.

18 Lemoine AN Jr, Rees TD, Newell FW. Discussion. Plast Reconstr Surg 1973; 52: 12-5.

19 Wiggs EO. Blepharoplasty complications. Trans Am Acad Ophthalmol Otolaryngol 1976; 81: OP603-6.

20 Moser MH, DiPirro E, McCoy FJ. Sudden blindness following blepharoplasty: report of seven cases. Plast Reconstr Surg 1973; 51 : 364-70. 